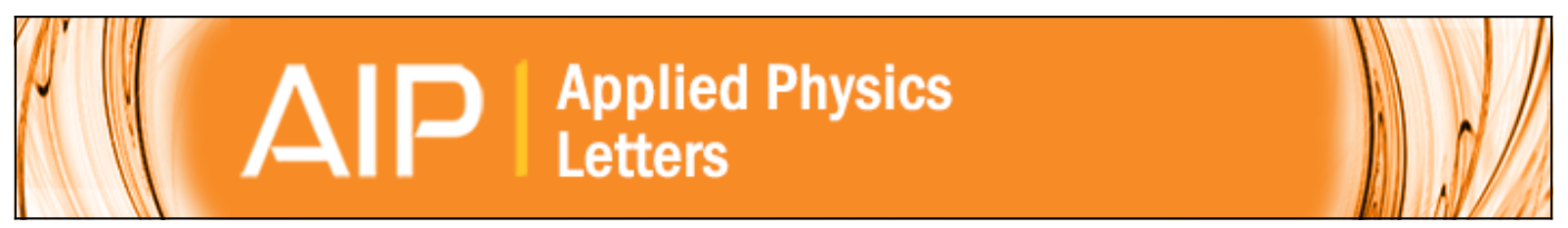

\title{
Pure nonlinear optical activity in metamaterials
}

llya V. Shadrivov

Citation: Applied Physics Letters 101, 041911 (2012); doi: 10.1063/1.4739849

View online: http://dx.doi.org/10.1063/1.4739849

View Table of Contents: http://scitation.aip.org/content/aip/journal/apl/101/4?ver=pdfcov

Published by the AIP Publishing

\section{Articles you may be interested in}

Active control of chirality in nonlinear metamaterials

Appl. Phys. Lett. 106, 091109 (2015); 10.1063/1.4914343

Complementary chiral metamaterials with giant optical activity and negative refractive index

Appl. Phys. Lett. 98, 161907 (2011); 10.1063/1.3574909

Second-harmonic generation and spectrum modulation by an active nonlinear metamaterial Appl. Phys. Lett. 94, 134102 (2009); 10.1063/1.3111437

Optical activity in extrinsically chiral metamaterial

Appl. Phys. Lett. 93, 191911 (2008); 10.1063/1.3021082

Strong optical activity in chiral metamaterials of metal screw hole arrays

Appl. Phys. Lett. 89, 211105 (2006); 10.1063/1.2392787

\section{Horizon"OPO}

Tunable power and performance

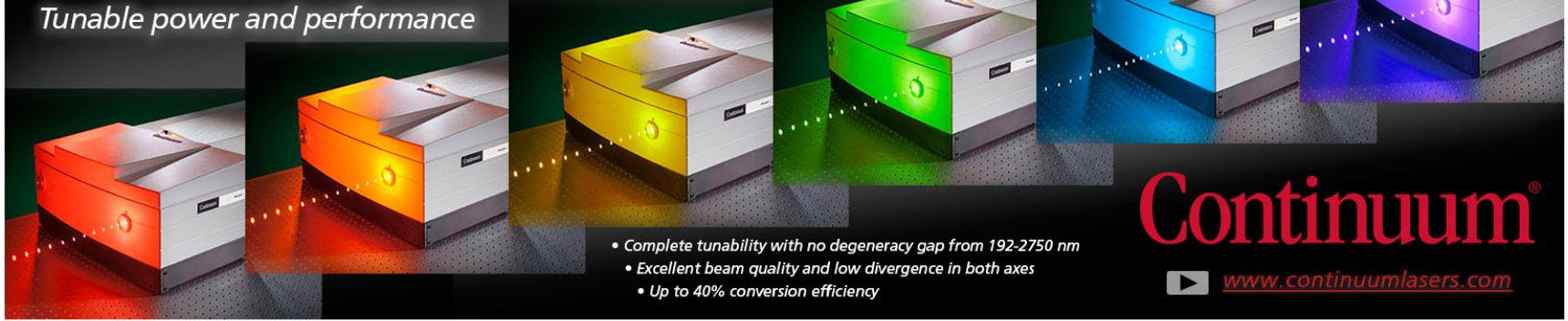




\title{
Pure nonlinear optical activity in metamaterials
}

\author{
Ilya V. Shadrivov \\ Nonlinear Physics Centre, Research School of Physics and Engineering, Australian National University, \\ Canberra ACT 0200, Australia
}

(Received 7 June 2012; accepted 16 July 2012; published online 27 July 2012)

\begin{abstract}
We demonstrate a type of meta-atom for creating metamaterials with giant nonlinear optical activity but vanishing linear optical activity in a wide frequency range. Such properties are not found in any natural materials, and we call this regime as pure nonlinear optical activity. We further extend our design concept and show that the metamaterial can be tuned dynamically to exhibit either positive or negative polarization rotation. (c) 2012 American Institute of Physics. [http://dx.doi.org/10.1063/1.4739849]
\end{abstract}

Electromagnetic metamaterials are designed to have properties not found in natural materials. For example, it was shown that one can achieve artificial magnetism at optical frequencies, negative refraction, enhanced chirality, and optical activity. ${ }^{1-4}$ Importantly, metamaterials allow separate engineering of electric, magnetic, as well as bianisotropic properties, including chirality. Chiral composite structures demonstrate giant optical activity near the resonance of meta-atoms, ${ }^{4}$ which is a metamaterial analogue of the Cotton effect. It is worth mentioning that artificial chiral composite structures were actively studied for several decades, with the main focus of earlier research on their absorbing properties (see, e.g., Refs. 5 and 6 and references therein).

Chiral object is the one which cannot be superimposed with its mirror image. A material made of chiral molecules or artificial particles transmits right- and left-handed circular polarized waves with different velocities. As a result, linearly polarized electromagnetic wave changes its polarization as it propagates in such medium, and this is called linear optical activity or gyrotropy. When the angle of the rotation of the plane of polarization depends on the intensity of the wave, the effect is called nonlinear optical activity or nonlinear gyrotropy. ${ }^{7}$ In natural crystals, the nonlinear optical activity is practically negligible and it is hard to detect on the background of linear optical activity. Metamaterials, however, can be designed to have large values of the nonlinear gyrotropy in various frequency ranges. ${ }^{8,9}$

In this letter, we propose a metamaterial which chirality can be dynamically induced in a non-chiral medium. This becomes possible in a racemic mixture containing equal amounts of right- and left-handed spirals, in which one type of spirals is either tunable or nonlinear. We demonstrate this effect numerically and experimentally for the example of electromagnetic waves scattering on a pair of canonical spirals of different handedness, where one of the spirals contains a nonlinear element. We show that initially nonoptically active pair exhibits polarization rotation once we increase the wave intensity. In this regime, the linear optical activity is negligible, while the nonlinear contribution is significant. We call this effect pure nonlinear optical activity. To the best of our knowledge, there are no natural materials with such properties. Additionally, we propose a design of meta-atom which optical activity changes its sign as we tune the control element.
Our meta-atom consists of a pair of canonical spirals ${ }^{10}$ as shown in Fig. 1. Without tunable element such a pair has mirror symmetry, and it does not exhibit any chiral response. Once we modify one of the spirals, we destroy the symmetry, and the pair becomes chiral. We study two configurations. First, balanced configuration is when both spirals have inclusions: one spiral has linear inclusion and another spiral has nonlinear inclusion. We choose the lumped element inclusions so that their impedances in the linear regime coincide, and the structure is mirror symmetric. The linear optical activity of the two spirals vanishes in such a regime. In the second, unbalanced case, we start with asymmetric structure, when only nonlinear element is inserted into one of the spirals, and therefore, the mirror symmetry is violated already in the linear regime.

We perform numerical simulations of the structure where both spirals have inclusions. We use CST Microwave Studio, and simulate electromagnetic wave propagation in a circular waveguide of the diameter of $59.7 \mathrm{~mm}$ corresponding to our experimental setup. The spirals are made of $0.16 \mathrm{~mm}$ thick wire. Length of the arms is $l=13 \mathrm{~mm}$, and the loop radius is $R=3 \mathrm{~mm}$. To model varactor diode that we plan to use in the experiment, we insert a lumped element having serial RLC circuit model into each spiral, with $R=0.8 \Omega, L=0.01 \mathrm{pH}$, and $\mathrm{C}$ fixed in one spiral at $2.7 \mathrm{pF}$, while in the second (control) spiral, the capacitance $C$ is

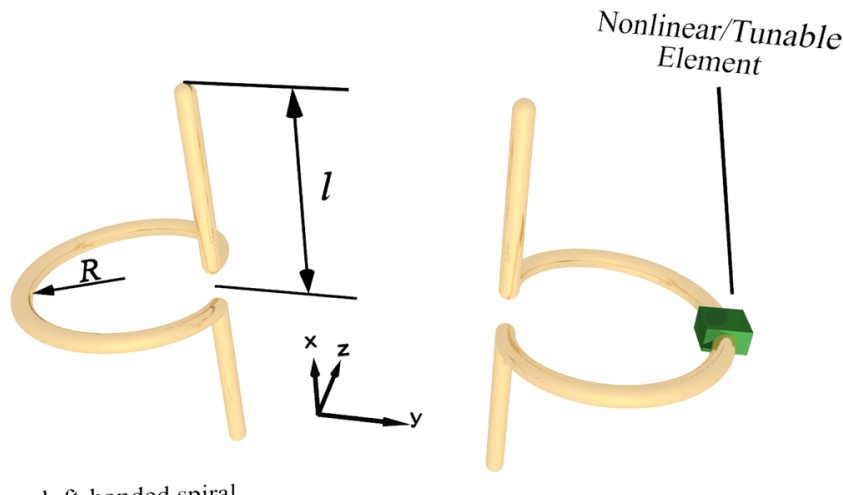

left-handed spiral

right-handed spiral

FIG. 1. Pair of canonical spirals with different handedness. Insertion of a nonlinear or tunable element into one of the spirals allows inducing and controlling the chiral response of such a pair of resonators. 
changing from $2.7 \mathrm{pF}$ to $2 \mathrm{pF}$, mimicking the self-induced tunability in a varactor diode. ${ }^{11}$ Spirals are placed perpendicular to the axis of the waveguide with their vertical parts separated by $30 \mathrm{~mm}$. Results of our simulations are shown in the Fig. 2 in the form of circular dichroism and polarization rotation as a function of frequency. We clearly see, that when the capacitance of the control element coincides with that in the passive spiral (at $2.7 \mathrm{pF}$ ), the optical activity is almost absent, while for $\mathrm{C}=2 \mathrm{pF}$, we observe significant circular dichroism and polarization rotation. Small optical activity observed in the fully symmetric case can be attributed to the numerical error due to a non-symmetric mesh generated by the software.

To test our ideas experimentally, we prepared a sample containing two canonical spirals made of a $0.16 \mathrm{~mm}$ thick copper wire with the same sizes as those used in numerical simulations. Here, we focus on the ability to dynamically tune the response of one of the spirals using nonlinear varactor diodes. ${ }^{11}$ The right-handed spiral is cut in the middle of the loop and the varactor diode (Skyworks 1405-079) is soldered to the wires. To mimic the response of the varactor diode in the linear regime, another spiral is also cut, and we soldered $2.7 \mathrm{pF}$ chip capacitor and $1 \Omega$ resistor into the loop. The spiral with nonlinear element resonates around $3.46 \mathrm{GHz}$, and the linear spiral is fine tuned by cutting its length to resonate at the same frequency. After tuning, both spirals are fixed to the styrofoam holder with their axis parallel; the distance between vertical sections of spirals is $30 \mathrm{~mm}$.

Experiments are performed in the $3-5 \mathrm{GHz}$ frequency range using a vector network analyzer (VNA, Rohde and Schwartz ZVB20), microwave amplifier (model HP $83020 \mathrm{~A}$ ), and a microwave waveguide polarimeter. The po-
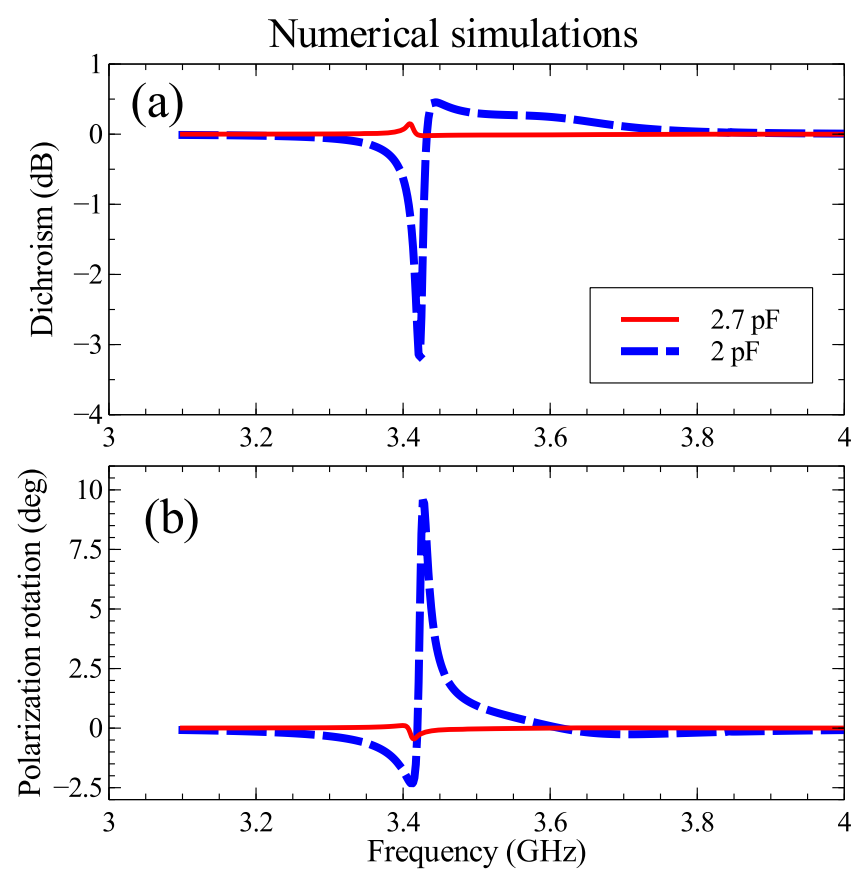

FIG. 2. Circular dichroism (a) and polarization rotation (b) calculated for a pair of spirals with resonant lumped element inclusions. The spirals are placed in the plane perpendicular to the axis of the waveguide, with the wave propagating in $z$-direction (see Fig. 1). Solid-lumped capacitances in both spirals are $2.7 \mathrm{pF}$; dashed-capacitance of the right-handed (control) spiral is $2 \mathrm{pF}$, while left-handed spiral contains $2.7 \mathrm{pF}$ capacitor. larimeter contains excitation part made of a coaxial-to-waveguide adapter, rectangular waveguide (W229), rectangularto-circular waveguide converter, and a piece of the circular waveguide. This structure excites either left-handed or righthanded circular polarized wave depending on the type of the rectangular-to-circular converter used. The analyzing part of the polarimeter is identical to the excitation part. Using different permutations of the polarizer/analyzer, we measure complete transmission matrix. In order to obtain the transmission coefficients, we divide each respective transmitted signal by the incident signal measured using $-22 \mathrm{~dB}$ directional coupler placed at the input of the polarimeter. To eliminate the contribution of the polarimeter components, the measurements are normalized to the corresponding transmission coefficients of the empty waveguide. The crucial part of high power measurements is power calibrations, which ensure that the amplitudes of the waves of different frequencies remain constant during the spectral scan. In our experiments, the calibration is performed for each power level using Rohde and Schwarz power sensor (model Z23) connected to a $-22 \mathrm{~dB}$ directional coupler placed before the polarimeter.

We measure the properties of the spirals in a circular waveguide section of the polarimeter. We place the spirals so that their arms are in the plane perpendicular to the waveguide axis. This corresponds to the wave propagation along $z$-axis on the Fig. 1. We measure transmission of the right-handed circularly polarized waves $T_{++}$, left-handed waves $T_{--}$, and corresponding polarization conversion coefficients $T_{+-}$and $T_{-+}$. The dichroism is found as the ratio $\left|T_{++} / T_{--}\right|$, while the polarization rotation is $\Delta \Theta$ $=\left[\arg \left(T_{++}\right)-\arg \left(T_{--}\right)\right] / 2$. Dependence of these parameters on frequency measured for low and high powers of the

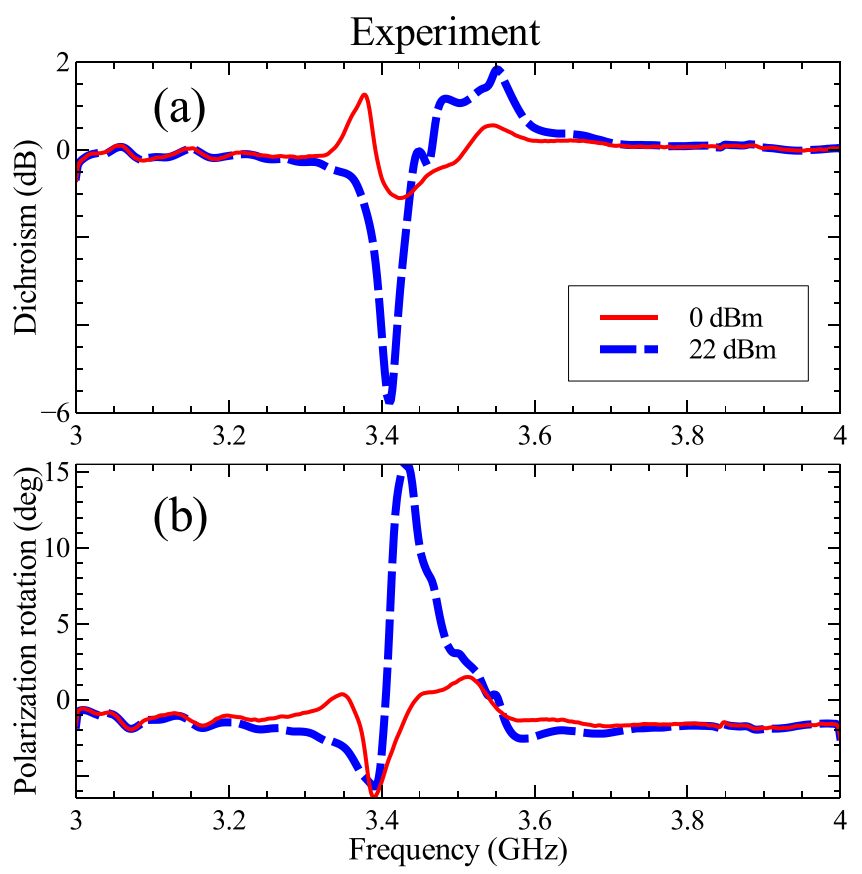

FIG. 3. Experimentally measured (a) dichroism and (b) polarization rotation by a pair of spirals, each having a lumped element included into the loop. The spirals are placed in the plane perpendicular to the axis of the waveguide, with the wave propagating in $z$-direction (see Fig. 1). Solid-low power, dashed-high power. 
incident wave is shown in Fig. 3. We observe a good agreement with predictions of our numerical simulations, with the polarization rotation angle at the frequencies close to the resonance being much larger at high powers than in the linear regime. The finite polarization rotation in the low power regime appears because the resonance response of the linear spiral does not exactly coincide with that of the nonlinear spiral. We successfully matched resonant frequencies of the spirals; however, the quality factors of the resonances were still slightly different.

Our measurements allow us to calculate the nonlinear gyrotropy of the structure as $N G=\Delta \Theta /(\Delta I h)$, where $\Delta I=\Delta P / S$ is the energy flow, $P$ is the input power, $S$ is the crosssection area of the waveguide, and $h$ is effective thickness of the sample. In further calculations, we use the physical sample thickness of $6.4 \mathrm{~mm}$. The nonlinear gyrotropy as a function of frequency is shown in Fig. 4, and it even exceeds that previously reported for the pair of wires. ${ }^{8}$ We see that our structure exhibits giant nonlinearly induced optical activity, while linear optical activity vanishes, and we refer to this regime as pure nonlinear optical activity.

Next, we study the structure, in which only one of the spirals contains nonlinear lumped inclusion, while another spiral does not have any lumped elements (unbalanced case). We have taken slightly shorter canonical spirals with the arm length of $l=10 \mathrm{~mm}$. As expected, already in linear regime, the structure shows significant resonant optical activity, as shown in Fig. 5. This resonant response shifts to higher frequency with power. As a result, the polarization rotation and dichroism are strongly power-dependant in the corresponding frequency range. As an example, at $4.15 \mathrm{GHz}$, the polarization rotation angle changes from approximately $-4^{\circ}$ to $+9^{\circ}$ with the change of incident power from $0 \mathrm{dBm}$ to $22 \mathrm{dBm}$. Such dependence of the polarization rotation on power corresponds to the giant nonlinear gyrotropy. ${ }^{8,9}$ At a single frequency around $4.1 \mathrm{GHz}$, we also observe remarkable nonlinear gyrotropy, but in the linear regime, the optical activity vanishes. This is the regime of pure nonlinear optical activity similar to the previously considered balanced case, with the difference that in this unbalanced structure, it appears only in an extremely narrow band, whereas in balanced case, the effect is broadband. It is worth mentioning that one can achieve somewhat similar effect by using composite structure with single type of chiral inclusions. Indeed,

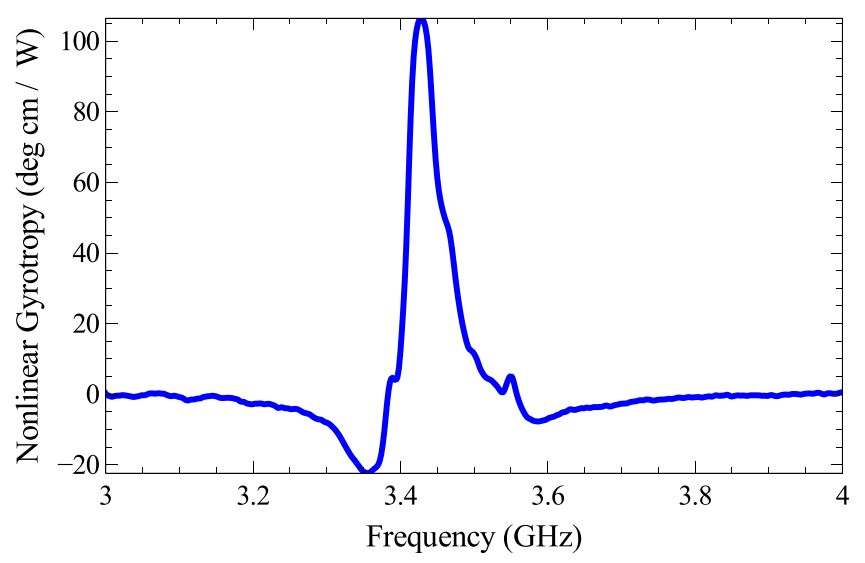

FIG. 4. Nonlinear gyrotropy of the pair of canonical spirals.
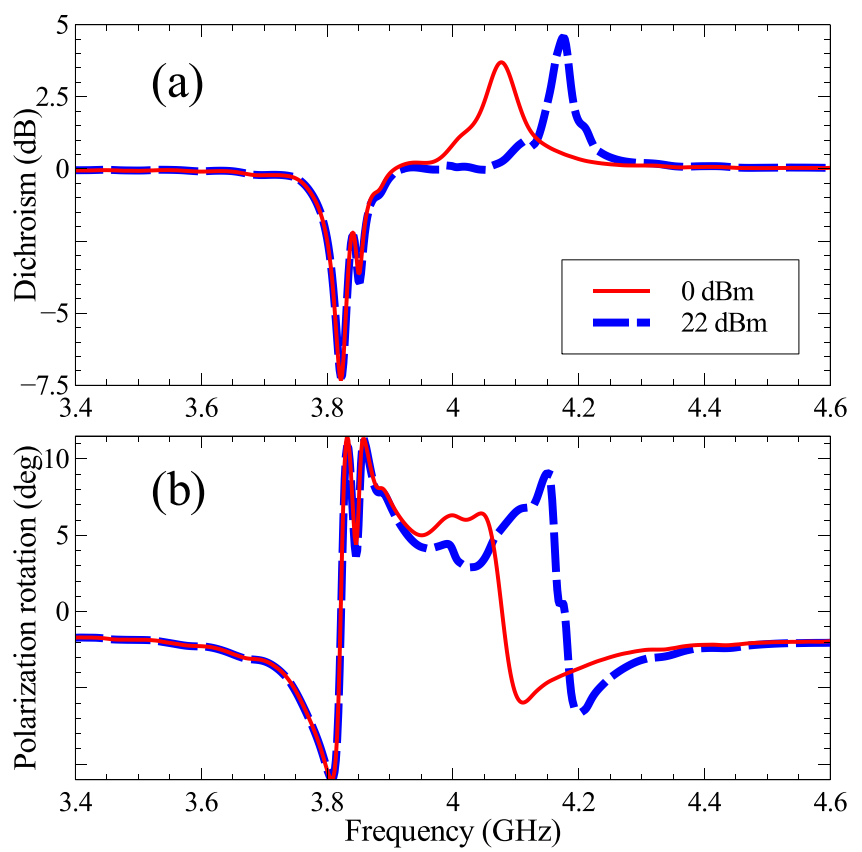

FIG. 5. Same as in Fig. 3 but for a pair of unbalanced spiral resonators.

such composite exhibits Cotton-like optical activity near the resonance, i.e., polarization rotation changes sign at the frequency of maximum absorption. Now if we employ the nonlinearity or tunability concept, by shifting the resonance ${ }^{8}$ of the meta-atoms, we are able for a given frequency not only to change the value of the optical activity, but we can also change its sign. This effect, however, does not allow broadband vanishing linear optical activity as it is possible in a racemic mixture of the spiral inclusions suggested in the form of balanced structure. This conclusion once again underlines the unique type of the response of the balanced racemic mixture of chiral inclusions showing pure nonlinear optical activity.

We would like to mention that similar approach can be used for creating the nonlinearity-induced broadband suppression of the optical activity. In order to achieve this, one would design the left- and right-handed chiral components of the composite structure so that their resonant frequencies (and quality factors of the resonances) would coincide at high power. As a result, at low power the polarization rotation will be significant near the resonances; however, for high power, the structure will become racemic, and the optical activity will vanish.

In conclusion, we have proposed a new approach for creating a chiral structure with tunable response, when the chirality can be nonlinearly induced by incident electromagnetic wave. We demonstrated a new regime of pure nonlinear optical activity in the composite structure which is non-chiral for low signal intensities. In the unbalanced structure with spirals of different handedness, which have different resonant properties in linear regime, we observe that the polarization rotation can change its sign as we change the intensity of the electromagnetic wave. Realization of large nonlinear gyrotropy in the optical frequency range, ${ }^{9}$ suggests that our concept can potentially be applied for higher frequencies. 
Author wishes to thank D. A. Powell and Yu. S. Kivshar for useful discussions. The work was supported by the Australian Research Council through Discovery Projects scheme.

${ }^{1}$ V. G. Veselago, Sov. Phys. Usp. 8, 2854 (1967) [Sov. Phys. Usp. 10, 509 (1968)].

${ }^{2}$ J. B. Pendry, Phys. Rev. Lett. 85, 3966 (2000).

${ }^{3}$ R. A. Shelby, D. R. Smith, and S. Schultz, Science 292, 77 (2001).

${ }^{4}$ A. V. Rogacheva, V. A. Fedotov, A. S. Schwanecke, and N. I. Zheludev, Phys. Rev. Lett. 97, 177401 (2006).
${ }^{5}$ R. Ro, V. V. Varadan, and V. K. Varadan, IEE Proc., Part H 139, 441 (1992).

${ }^{6}$ A. Lakhtakia, V. K. Varadan, and V. V. Varadan, J. Mater. Res. 8, 917 (1993).

${ }^{7}$ Y. P. Svirko and N. I. Zheludev, Polarization of Light in Nonlinear Optics (Wiley, New York, 1998).

${ }^{8}$ I. V. Shadrivov, V. A. Fedotov, D. A. Powell, Yu. S. Kivshar, and N. I. Zheludev, New J. Phys. 13, 033025 (2011).

${ }^{9}$ M. Ren, E. Plum, J. Xu, and N. I. Zheludev, Nat. Commun. 3, 833 (2012).

${ }^{10}$ E. Saenz, I. Semchenko, S. Khakhomov, K. Guven, R. Gonzalo, E. Ozbay, and S. Tretyakov, Electromagnetics 28, 476 (2008).

${ }^{11}$ D. A. Powell, I. V. Shadrivov, Yu. S. Kivshar, and M. V. Gorkunov, Appl. Phys. Lett. 91, 144107 (2007). 